\title{
REAÇÃO LEUCEMÓIDE ASSOCIADA À SARCOMA INDIFERENCIADO EM INTESTINO DELGADO DE CÃO
}

\section{(Extreme neutrophilic leucocytosis associated with intestinal sarcoma in a dog)}

\author{
DITTRICH, R.L. ${ }^{\text {; }}$ RODASKI, S. ${ }^{1}$; PLUGGE, N.F.; ALVARES, A.A.A. \\ SPREA, G. ${ }^{3}$; ZANETTI, M.B.F.'; CARMO, F.S. ${ }^{2}$ \\ 'Departamento de Medicina Veterinária-UFPR; \\ ${ }^{2}$ Hospital Veterinário-UFPR; \\ ${ }^{3}$ Curso de Pós-Graduação em Ciências Veterinárias-UFPR.
}

\begin{abstract}
RESUMO - Um cão da raça Poodle, de 11 anos de idade foi encaminhado ao Hospital Veterinário da Universidade Federal do Paraná (UFPR), com histórico de hiporexia e emagrecimento há seis meses. No exame físico constatou-se caquexia, mucosas hipocoradas e ausência de febre. No hemograma observou-se anemia, leucocitose, neutrofilia extrema e desvio nuclear dos neutrófilos à esquerda (101.200 leucócitos/ $\mu \mathrm{L} ; 87.032$ segmentados/ $\mu \mathrm{L} ; 4.048$ bastonetes $/ \mu \mathrm{L}$ ). No exame da medula óssea observou-se hiperplasia mielóide sem evidências de leucemia, confirmando a ocorrência de reação leucemóide. A ultra-sonografia abdominal identificou estrutura de 3,43 cm em alças intestinais, sugestiva de neoplasia mural de segmento piloro-duodenal. Realizou-se a exérese cirúrgica. O tumor foi identificado como neoplasia maligna de origem mesenquimal no exame histopatológico. No pós-operatório observou-se apetite normal do cão e a resolução das anormalidades hematológicas ( 6.348 neutrófilos/ $\mu \mathrm{L} ; 0$ bastonetes $/ \mu \mathrm{L})$. O presente trabalho descreve um caso de reação leucemóide paraneoplásica com resolução das anormalidades após a remoção da neoplasia.
\end{abstract}

Palavras-chaves: reação leucemóide paraneoplásica; cão; mielograma.

ABSTRACT - An eleven-year-old Poodle was carried to the Veterinary Hospital of the Universidade Federal do Paraná (UFPR) with a six-month history of weight loss and hiporexia. On physical examination the dog shows afebrile and had pale mucous membranes. Routine haematological examination revealed anemia, leucocytosis, marked mature neutrophilia left shift $(101,200 \mathrm{cell} / \mu \mathrm{L}$; 87,032 segmented $/ \mu \mathrm{L} ; 4,048 \mathrm{band} / / \mu \mathrm{L}$ ). Examination of bone marrow to the smears confirmed a marked leukemoid response, indicating myeloid hyperplasia without evidence of leukemia. Imagery showed a presence of a 3,43 cm diameter mass in the piloro-duodenal segment. The mass was surgically removed. Histologically, it revealed a malignancy suggestive of sarcoma. Post-operatively, the dog's appetite improved. On re- evaluation, routine haematology revealed a reduction in the mature and band neutrophils count $(6,348$ neutrophils $/ \mu \mathrm{L} ; 0$ band $/ \mu \mathrm{L})$. This report describes a case of paraneoplastic leukemoid reaction with resolution of the abnormality following removal of the neoplasm.

Key-words: paraneoplastic leukaemoid reaction; dog; bone marrow aspirate. 


\section{Introdução}

As principais causas de leucocitose e neutrofilia incluem a resposta por glicocorticóides ou por catecolaminas, a inflamação e as neoplasias. A leucocitose neutrofílica extrema em cães pode ocorrer principalmente nas neoplasias, nasdoenças infecciosas, nas doenças imunomediadas, na necrose tecidual e na leucemia mielóide. Segundo MESSICK, 2000; LUCROY e MADEWELL, 2001, as causas de leucocitose e neutrofilia extremas em cães e gatos devem ser investigadas devido a sua associação com elevadas taxas de mortalidade. Reação leucemóide é uma leucocitose extrema, geralmente associada com neutrofilia e acentuado desvio nuclear dos neutrófilos à esquerda. Em alguns animais com neoplasias malignas e benignas ocorre a reação leucemóide paraneoplásica. Nos casos de carcinomas em cães, foram observados maior incidência de reação leucemóide (KNOTTENBELT et al., 2000; SQUIRES e THOMPSON, 2004).

O objetivo deste trabalho é descrever o diagnóstico da causa de leucocitose neutrofílica extrema em um cão com hiporexia e emagrecimento progressivo, atendido no Hospital Veterinário da Universidade Federal do Paraná.

\section{Material e Métodos}

Um cão macho, da raça Poodle, de 11 anos de idade foi atendido no Hospital Veterinário da Universidade Federal do Paraná (HV-UFPR) com histórico de hiporexia e emagrecimento há seis meses. No exame físico constatou-se caquexia, mucosas hipocoradas e ausência de febre.

Foram solicitados exames complementares que incluíram hemogramas, urinálises, bioquímicos de avaliação hepática (enzima alanina amino transferase - ALT), renal (creatinina), pancreática (lipase e amilase), determinação da proteína plasmática total (PPT) e do fibrinogênio. Os hemogramas foram solicitados nas três consultas do paciente, sendo considerado o dia zero (0) como a primeira consulta e os dias 21 e 35 como a segunda e terceira consultas, respectivamente.
Os exames de radiografia torácica, ultrasonografia abdominal, contagem de plaquetas e punção de medula óssea foram solicitados na terceira consulta do paciente.

As amostras de sangue foram obtidas por venopunção e acondicionadas em tubos com anticoagulante (EDTA 10\%) para realização dos hemogramas, e sem anticoagulante para obtenção do soro e determinação dos parâmetros bioquímicos. Os hemogramas, a proteína plasmática total (PPT) e o fibrinogênio foram realizados segundo JAIN (1993). As análises bioquímicas foram determinadas em sistema bioquímico semi-automático CELM SBA-200.

As amostras de urina para urinálise foram obtidas por cistocentese. A punção de medula óssea foi realizada em crista da tíbia, com agulha hipodérmica $25 \times 8$. Procedeu-se a extensão em lâmina, coloração com Wright e análise em microscopia óptica.

Foram realizadas radiografia torácica em posicionamento latero-lateral e ventro-dorsal e ultra-sonografia abdominal com "doppler" colorido como auxílio à conduta diagnóstica e terapêutica.

\section{Resultados}

Os resultados das análises hematológicas e da proteína plasmática total estão na TABELA 1.

No primeiro hemograma constatou-se anemia severa microcítica e hipocrômica, acentuada leucocitose com neutrofilia, linfocitose, eosinofilia e monocitose. $\mathrm{Na}$ urinálise e na avaliação radiográfica do tórax não foram observadas alterações. Os parâmetros bioquímicos estavam dentro dos valores de referência.

O protocolo terapêutico inicial foi com enrofloxacina (5 $\mathrm{mg} / \mathrm{Kg} / \mathrm{BID})$. No retorno verificou-se persistência da hiporexia, vômitos esporádicos, um episódio de síncope durante esforço físico e posicionamento de prece freqüente. A avaliação clínica do paciente demonstrou intensa sensibilidade abdominal, desidratação moderada e persistência da caquexia e mucosas hipocoradas, efetivandose internamento do paciente. 
Reação leucemóide associada à sarcoma indiferenciado em intestino delgado de cão

TABELA 1 - HEMOGRAMAS DE UM CÃO COM LEUCOCITOSE NEUTROFÍLICA EXTREMA ASSOCIADA A SARCOMA INDIFERENCIADO EM INTESTINO DELGADO. CURITIBA - 2005.

\begin{tabular}{|c|c|c|c|c|c|c|c|c|c|c|}
\hline & \multicolumn{2}{|c|}{ Dia 0} & \multicolumn{2}{|c|}{ Dia 21} & & Dia 35 & \multicolumn{2}{|c|}{$\begin{array}{c}54 \text { dias após } \\
\text { a cirurgia }\end{array}$} & \multicolumn{2}{|c|}{$\begin{array}{l}\text { Valores de } \\
\text { Referência* }\end{array}$} \\
\hline ERITROGRAMA & \multirow{2}{*}{\multicolumn{2}{|c|}{4,36}} & \multirow{2}{*}{\multicolumn{2}{|c|}{2,91}} & \multirow{2}{*}{\multicolumn{2}{|c|}{2,51}} & \multirow{2}{*}{\multicolumn{2}{|c|}{5,85}} & \multirow{2}{*}{\multicolumn{2}{|c|}{5,5 a 8,5}} \\
\hline $\begin{array}{l}\text { Eritrócitos } \\
\text { (milhões/ } / \mu \mathrm{L} \text { ) }\end{array}$ & & & & & & & & & & \\
\hline $\mathrm{Ht}(\%)$ & \multirow{2}{*}{\multicolumn{2}{|c|}{$\begin{array}{c}19 \\
6.00\end{array}$}} & \multicolumn{2}{|r|}{20} & \multicolumn{2}{|r|}{18} & \multicolumn{2}{|r|}{38} & \multicolumn{2}{|r|}{37 a 55} \\
\hline $\mathrm{Hb}(\mathrm{g} / \mathrm{dL})$ & & & \multirow{2}{*}{\multicolumn{2}{|c|}{$\begin{array}{c}6,20 \\
68,73\end{array}$}} & \multirow{2}{*}{\multicolumn{2}{|c|}{$\begin{array}{c}5,40 \\
71,70\end{array}$}} & \multicolumn{2}{|r|}{14,9} & \multicolumn{2}{|r|}{12 a 18} \\
\hline VGM $(\mu \mathrm{m} 3)$ & \multicolumn{2}{|c|}{43,58} & & & & & \multirow{2}{*}{\multicolumn{2}{|c|}{$\begin{array}{l}65,6 \\
393\end{array}$}} & \multicolumn{2}{|r|}{60 a 77} \\
\hline CHGM (\%) & \multicolumn{2}{|c|}{31,58} & \multicolumn{2}{|r|}{31,00} & \multicolumn{2}{|r|}{30,00} & & & \multicolumn{2}{|r|}{32 a 36} \\
\hline $\begin{array}{l}\text { Observações } \\
\text { LEUCOGRAMA }\end{array}$ & \multicolumn{2}{|c|}{$\mathrm{a} / \mathrm{b}$} & \multicolumn{2}{|r|}{$\mathrm{a} / \mathrm{b}$} & \multicolumn{2}{|r|}{$\mathrm{a} / \mathrm{b} / \mathrm{c}$} & \multicolumn{2}{|r|}{-} & & - \\
\hline Leucócitos $(/ \mu \mathrm{L})$ & \multicolumn{2}{|c|}{88.100} & \multicolumn{2}{|c|}{101.200} & \multicolumn{2}{|c|}{91.000} & \multicolumn{2}{|c|}{9.200} & \multicolumn{2}{|c|}{6.000 a 17.000} \\
\hline & $\%$ & Abs & $\%$ & Abs & $\%$ & Abs & $\%$ & Abs & $\%$ & Abs \\
\hline Segmentados & 90 & 79.290 & 86 & 87.032 & 82 & 74.620 & 69 & 6.348 & $60-77$ & 3.000 a 11.500 \\
\hline Bastonetes & 0 & 0 & 4 & 4.048 & 4 & 3.640 & 0 & 0 & 0 a 3 & 0 a 300 \\
\hline Metamielócitos & 0 & 0 & 0 & 0 & 0 & 0 & 0 & 0 & 0 & 0 \\
\hline Linfócitos & 5 & 4.405 & 6 & 6.072 & 4 & 3.640 & 14 & 1.288 & 12 a 30 & 1.000 a 4.800 \\
\hline Eosinófilos & 2 & 1.762 & 1 & 1.012 & 1 & 910 & 16 & 1.472 & 2 a 10 & 100 a 1.250 \\
\hline Monócitos & 3 & 2.643 & 3 & 3.036 & 9 & 8.190 & 1 & 92 & 3 a 10 & 150 a 1.350 \\
\hline Basófilos & 0 & 0 & 0 & 0 & 0 & 0 & 0 & 0 & raros & raros \\
\hline Observações & & - & & - & & $d / e$ & & - & & - \\
\hline PPT (g/dL) & & 6,2 & & 5,0 & & 5,2 & & 6,2 & &, 0 a 8,0 \\
\hline
\end{tabular}

Ht. - hematócrito; Hb. - hemoglobina; Abs. - valores absolutos dos leucócitos; a - anisocitose;

b - policromatofilia (03/campo); c - poiquilocitose; d - neutrófilos tóxicos: picnose, cariorexe, granulações tóxicas e vacuolização citoplasmática; e - neutrófilos hipersegmentados; PPT - proteína plasmática total. *Valores de referência: JAIN, 1993.

No segundo hemograma constatou-se anemia normocítica e hipocrômica, leucocitose e neutrofilia extremas, com desvio nuclear de neutrófilos à esquerda (DNNE) regenerativo, linfocitose, monocitose e hipoproteinemia.

Durante o período de internamento foram realizadas fluidoterapia, antibioticoterapia e analgesia. $O$ antimicrobiano ceftriaxona $(15 \mathrm{mg} /$ $\mathrm{Kg} / \mathrm{BID}$ ) foi associado à enrofloxacina. Para alívio da dor foi instituído $\mathrm{N}$-butil-brometo de hioscina com dipirona (25 mg/Kg/BID) e cloridrato de tramadol (2 $\mathrm{mg} / \mathrm{Kg} / \mathrm{BID})$.

O resultado do mielograma está apresentado na TABELA 2. Observou-se hiperplasia mielóide, com relação mielóide-eritróide $(M / E)$ de 9,5/1. As células apresentaram morfologia normal. Foram observados $82,73 \%$ de neutrófilos segmentados e bastonetes, e alto índice de maturação mielóide, indicando um aumento da granulopoese com desvio à direita na medula óssea.

A ultra-sonografia abdominal revelou estrutura heterogênea em segmento intestinal, com diâmetro de $3,43 \mathrm{~cm}$, parede espessada focalmente, sem definição de seus folhetos e presença de gases em lúmen. Aárea pancreática não foi avaliada precisamente devido a proximidade da estrutura citada. A imagem verificada em abdome cranial direito foi sugestiva de neoplasia mural de segmento piloro-duodenal, sendo indicada a exérese cirúrgica.

No terceiro hemograma constatou-se anemia normocítica e hipocrômica, acentuada leucocitose, neutrofilia com DNNE regenerativo e monocitose. O número de plaquetas estava dentro dos valores de referência. $O$ animal permanecia com hipoproteinemia $(5,2 \mathrm{~g} / \mathrm{dL})$ e apresentou hiperfibrinogenemia de $600 \mathrm{mg} / \mathrm{dL}$ (valor de referência: $125-300 \mathrm{mg} / \mathrm{dL}$ ).

Realizou-se transfusão de sangue total (300 $\mathrm{mL})$ no período pré-operatório imediato. A exérese neoplásica constou de ressecção com $3,0 \mathrm{~cm}$ de margem de segurança, removendose assim 9,0 cm do íleo. Na seqüência, procedeu-se a enteroanastomose términoterminal, optando-se pelo padrão tipo "crushing" para a enterorrafia.

O tumor foi identificado como neoplasia maligna de origem mesenquimal (sarcoma indiferenciado) no exame histopatológico. 
TABELA 2 - MIELOGRAMA DE UM CÃO COM LEUCOCITOSE NEUTROFÍLICA EXTREMAASSOCIADA A SARCOMA INDIFERENCIADO EM INTESTINO DELGADO. CURITIBA - 2005.

\begin{tabular}{lcc}
\hline \multirow{1}{*}{ Células } & \multicolumn{2}{c}{ Distribuição em Porcentagem (500 Células) } \\
\cline { 2 - 3 } & $\begin{array}{c}\text { Valores De } \\
\text { Referência* }(\%)\end{array}$ & $\begin{array}{c}\text { Cão Com Leucocitose Neutrofílica } \\
\text { Paraneoplásica }\end{array}$ \\
\hline Série Eritrocítica & 0,2 & 0,61 \\
Rubroblastos & 3,9 & 0,48 \\
Pró-Rubrócitos & 27,0 & 1,71 \\
Rubrócitos & 15,3 & 6,73 \\
Metarubrócitos & 46,4 & 9,53 \\
Total & & \\
Série Granulocítica & 0,0 & 0,24 \\
Mieloblastos & 1,3 & 1,71 \\
Pró-Mielócitos & 9,0 & 1,34 \\
Mielócito Neutrófilo & 0,0 & 0,61 \\
Mielócito Eosinófilo & & 0,24 \\
Mielócito Basófilo & 7,5 & 2,44 \\
Metamielócito Neutrófilo & 2,4 & 0,12 \\
Metamielócito Eosinófilo & 13,6 & 38,43 \\
Neutrófilo Bastonete & 0,9 & 0,31 \\
Eosinófilo Bastonete & 18,4 & 44,30 \\
Neutrófilo Segmentado & 0,3 & 0,73 \\
Eosinófilo Segmentado & 0,0 & 0,0 \\
Basófilo Segmentado & 53,4 & 90,47 \\
Total & $1,4: 1,0$ & $9,5: 1,0$ \\
Relação M/E & & \\
Outras Células & 0,0 & 0,0 \\
Pró-Linfócitos & 0,2 & 0,0 \\
Linfócitos & 0,0 & 0,0 \\
Monócitos & &
\end{tabular}

*Valores de referência: JAIN, 1993.

Após 30 dias do procedimento cirúrgico o paciente retornou ao Hospital Veterinário com grande melhora da condição clínica, apresentando normalização do apetite, ganho de peso $(3 \mathrm{Kg})$, mucosas normocoradas e comportamento ativo. Não foram mais observados posição de alívio, desconforto abdominal e vômitos. O hemograma realizado 54 dias após a exérese do tumor não apresentou alterações.

\section{Discussão}

As causas de leucocitose neutrofílica são os agentes infecciosos, necrose tecidual, doenças imuno-mediadas, corticosteróides endógenos ou exógenos, catecolaminas, neoplasia hematopoiética e a neutrofilia paraneoplásica (STOCKHAM et al., 2003).

A magnitude da neutrofilia observada no cão deste relato foram inconsistentes com leucocitose induzida por catecolaminas e por corticosteróides, que são limitadas. Na resposta às catecolaminas ocorre a neutrofilia fisiológica, que nos cães não excede duas vezes o limite superior de referência (STOCKHAM et al., 2003). Os valores de neutrófilos observados nos três leucogramas deste cão, de 79.290, 87.032 e 74.620 neutrófilos segmentados $/ \mathrm{mL}$, respectivamente, foram muito superiores aos valores relatados por resposta aos corticosteróides, que induzem nos cães uma neutrofilia de no máximo três vezes o limite superior de referência (LUCROY e MADEWELL, 1999; STOCKHAM et al., 2003). No exame físico o paciente não apresentou sinais de trauma ou necrose tecidual, descartando-se estes fatores como causas da leucocitose e neutrofilia.

No presente caso, as doenças infecciosas foram desconsideradas devido aos fatores como persistente ausência de febre e os resultados das avaliações radio e ultrasonográficas. A utilização prolongada de 
agentes antimicrobianos de amplo espectro não apresentou resposta favorável. Devido aos sinais clínicos apresentados, principalmente o posicionamento de prece, houve a suspeita inicial de pancreatite. O diagnóstico não foi confirmado pelos exames bioquímicos e ultrasonográficos.

Nos três leucogramas do paciente foram observadas leucocitose com neutrofilia extremas e monocitose. No segundo e terceiro leucogramas foi constatado um desvio dos neutrófilos à esquerda de 4.048 e 3.640 bastonetes $/ \mathrm{mL}$, respectivamente. O termo reação leucemóide é utilizado para definir uma leucocitose extrema, geralmente associada com neutrofilia e acentuado desvio nuclear dos neutrófilos à esquerda. "Leucemóide" referese a semelhança do sangue com a leucemia mielóide crônica (LMC), contudo, a causa é o aumento da necessidade tecidual por neutrófilos e não uma doença mieloproliferativa (JAIN, 1993; STOCKHAM et al., 2003). Segundo LAPPIN e LATIMER (1988) e KNOTTENBELT et al. (2000), o número de leucócitos totais acima de $50.000 / \mathrm{mL}$ pode ser sugestivo de leucemia, porém, este valor de leucócitos e a presença de células com morfologia normal, geralmente estão associadas às doenças mais benignas e são denominadas de reação leucemóide. No entanto, LUCROY e MADEWELL (1999), observaram que cães com número de leucócitos totais igual ou superior a $50.000 / \mathrm{mL}$ e neutrofilia maior ou igual a $50 \%$, apresentaram alta taxa de mortalidade (62\%). No paciente deste estudo constatou-se monocitose, uma alteração que pode ocorrer nos casos de leucocitose paraneoplásica (DOLE et al., 2004).

Leucemias são neoplasias malignas do tecido hematopoiético e na maioria dos casos ocorre um aumento da célula sangüínea neoplásica no sangue periférico (ANDREWS, 2000; STOCKHAM et al., 2003). Na LMC observa-se leucocitose significativa com predomínio de neutrófilos segmentados e bastonetes. Em cães com LMC o número de leucócitos totais pode variar de 16.000 a $169.000 / \mathrm{mL}$, sendo na maioria neutrófilos segmentados. Em alguns casos, porém, ocorre o desvio à esquerda desordenado. A dificuldade do diagnóstico da LMC é a diferenciação da reação leucemóide secundária às infecções, doenças imuno-mediadas ou neoplasias. $\mathrm{Na}$ medicina humana, a presença do cromossoma Philadelphia é utilizada para diferenciar a LMC da reação leucemóide. No cão, anormalidades cromossomais específicas não foram relatadas (PERKINS, 2000; MESSICK, 2000).

A punção aspirativa da medula óssea foi realizada para caracterizar os elementos da medula e observar possíveis células anormais devido a leucocitose extrema do cão (> 90.000/ $\mathrm{mL}$ ). A avaliação da medula óssea também é utilizada para diferenciar a reação leucemóide da LMC (KNOTTENBELT et al., 2000). No cão do presente relato foram constatadas série eritrocítica de $9,53 \%$, série granulocítica de $90,47 \%$, número de células blásticas inferior a $30 \%(2 \%)$, relação $\mathrm{M}: \mathrm{E}>1$ (9,5:1), $82,73 \%$ de neutrófilos segmentados e bastonetes. Constatou-se o alto índice de maturação mielóide, indicando um aumento da granulopoese com desvio à direita na medula óssea. A medula óssea com desvio à direita é observada em pacientes com neutrofilia por resposta inflamatória ou infeciosa (JAIN, 1993). Os resultados do mielograma do cão foram compatíveis com hiperplasia mielóide devido ao número diminuído de células blásticas na medula óssea (2\%), neutrofilia periférica com valores de neutrófilos superiores a duas vezes o valor de referência, a ausência de alterações nas células da medula óssea e maturação ordenada (GRINDEM, 2000; MESSICK, 2000).

$\mathrm{Na}$ leucemia mielóide crônica a relação mielóide-eritróide $(\mathrm{M} / \mathrm{E})$ pode variar de 3:1 até 24:1 e ocorre geralmente uma maturação mielóide anormal e desordenada, com desvio à esquerda das células mielóides, ou seja, aumento do número de mieloblastos, prómielócitos e mielócitos neutrófilos (MESSICK, 2000). O mielograma do cão permitiu desconsiderar a leucemia mielóide crônica e indicar a ocorrência de uma hiperplasia mielóide.

Nos pacientes com neoplasias são observados vários sinais clínicos como conseqüência da invasão ou obstrução de estruturas e órgãos. De maneira indireta as neoplasias também causam diversos efeitos sistêmicos que são denominados de síndrome 
paraneoplásica (OGILVIE, 1997). As principais alterações associadas à síndrome paraneoplásica são anorexia, caquexia, anemia ou eritrocitose, leucocitose, trombocitopenia, hiper ou hipocalcemia, osteopatia hipertrófica, febre de origem desconhecida, hipoglicemia, hiperhistaminemia e hipergamaglobulinemia (YOUNG, 1997; MARCHETTI et al., 2005).

No paciente do presente estudo observouse hiporexia/anorexia, emagrecimento, caquexia e síncope durante esforço físico. A neoplasia localizava-se no intestino delgado, induzindo perdas nas funções de digestão e absorção de nutrientes. O peso normal do cão, de $10 \mathrm{Kg}$, diminuiu para $7 \mathrm{Kg}$ em seis meses. Trinta dias após a exérese cirúrgica da neoplasia o paciente havia recuperado seu escore corporal.

O animal apresentou anemia microcítica e hipocrômica com hipoproteinemia, possivelmente devido à perda inaparente de sangue intestinal e às dificuldades na digestão e absorção de nutrientes, também decorrentes do complexo anorexia/caquexia.

A identificação das anormalidades hematológicas neste cão, como leucocitose neutrofílica extrema, desvio à esquerda, monocitose, anemia, hiperplasia mielóide sem um foco de infecção, em associação ao diagnóstico da neoplasia e a resolução das anormalidades após a remoção do tumor, suportam o diagnóstico de reação leucemóide paraneoplásica.

Os casos de reação leucemóide neutrofílica são associados à vários tumores em humanos, como carcinomas, linfomas e sarcomas. Ocorre uma neutrofilia acentuada com ou sem monocitose que não podem ser atribuídas a uma resposta inflamatória ou a uma neoplasia hematopoiética primária. Em cães, as neoplasias benignas e malignas, principalmente carcinomas, podem estar associadas à leucocitose neutrofílica, como carcinoma renal, carcinoma pulmonar, adenocarcinoma tubular renal, pólipo retal adenomatoso e tumor retal. A neoplasia identificada neste paciente foi sarcoma indiferenciado, sendo que existem poucos relatos de sarcomas associados a reação leucemóide paraneoplásica em cães. Resultados similares foram observados nos casos de sarcoma histiocitário e fibrosarcoma (CHINN et al., 1985;
THOMPSON et al., 1992; SHARKEY et al., 1996; SQUIRES e THOMPSON, 2004).

Aleucocitose paraneoplásica ocorre porque as células neoplásicas produzem os fatores de crescimento hematopoiético, como o fator estimulador de colônias dos granulócitos (G-CSF) e fator estimulador de colônias dos granulócitosmacrófagos (GM-CSF), que foram identificados em cães e gatos. Estes fatores estimulam a medula óssea a aumentar a produção de neutrófilos, causando neutrofilia acentuada (GABRILOVE e GOLDE, 1993; SHARKEY et al, 1996; NAOM, 2001; DOLE et al., 2004).

Após a remoção do tumor, o cão apresentou melhora clínica e observou-se resolução das alterações hematológicas, indicando a ocorrência da síndrome paraneoplásica.

\section{Conclusões}

A leucocitose neutrofílica extrema deve ser investigada com exames complementares, devido a gravidade das possíveis causas. O monitoramento do paciente é necessário, com hemogramas seqüenciais e punção aspirativa da medula óssea, devido as dificuldades de diagnóstico e diferenciação da leucemia mielóide crônica. O sarcoma indiferenciado em intestino delgado de cão pode induzir a reação leucemóide paraneoplásica, e esta deve ser incluída na lista de diagnóstico diferencial para animais com neutrofilia extrema sem causa clínica aparente.

\section{REFERÊNCIAS}

ANDREWS, J.M. Classification and biology of myeloproliferative disorders. In: FELDMAN, B.F.; Zinkl, J.G.; Jain, N.C. Schalm's veterinary hematology. 5.ed. Philadelphia: Lippincott Williams \& Wilkins, 2000. p. 673-675.

CHINN, R.; MYERS, R.K.; MATTHEWS, J.A. Neutrophilic leukocytosis associated with metastatic fibrosarcoma in a dog. Journal of the American Veterinary Medical Association, Schaumburg, v. 18, p. 806-808, 1985.

DOLE, R.S.; MacPHAIL, C.M.; LAPPIN, M.R. Paraneoplastic leukocytosis with mature neutrophilia in cat with pulmonary squamous cell carcinoma. Journal of Feline Medicine and Surgery, London, v. 6, p. 391-395, 2004. 
Reação leucemóide associada à sarcoma indiferenciado em intestino delgado de cão

GABRILOVE, J.L.; GOLDE, D.W. Hematopoietic growth factors. In: De VITA, V.T, HILLMA, S., ROSEBER, A. S. Cancer: principles and practice of oncology. 4.ed. Philadelphia: JB Lippincott, 1993. p. $2275-2291$.

GRINDEM, C.B. Acute myeloid leukemia. In: FELDMAN, B.F.; ZINKL, J.G.; JAIN, N.C. Schalm's veterinary hematology. 5.ed. Philadelphia: Lippincott Williams \& Wilkins, 2000. p. 717-726.

JAIN, N.C. Essentials of veterinary hematology. Philadelphia: Lea and Febiger, 1993. 417 p.

KNOTTENBELT, C.M.; SIMPSON, J.W.; CHANDLER, M.L. Neutrophilic leucocytosis in a dog with a rectal tumour. Journal of Small Animal Practice, Oxford, v. 41, p. 457-460, 2000.

LAPPIN, M.R.; LATIMER, K.S. Hematuria and extreme neutrophilic leucocytosis in a dog with renal tubular carcinoma. Journal of the American Veterinary Medical Association, Schaumburg, v. 192, p. 1289-1292, 1988.

LUCROY, M.D; MADEWELL, B.R. Clinical outcome and associated diseases in dogs with leukocytosis and neutrophilia: 118 cases (1996-1998). Journal of the American Veterinary Medical Association, Schaumburg, v. 214, p. 805-807, 1999.

LUCROY, M.D; MADEWELL, B.R. Clinical outcome and diseases associated with extreme neutrophilic leukocytosis in cats: 104 cases (1991-1999). Journal of the American Veterinary Medical Association, Schaumburg, v. 218, p. 736-739, 2001.

MARCHETTI, V.; BENETTI, C.; CITI, S.; TACCINI, V. Paraneoplastic hypereosinophilia in a dog with intestinal T-cell lymphoma. Veterinary Clinical Patology, Santa Barbara, v. 34, p. 259-263, 2005.

MESSICK, J. B. Chronic myeloid leukemias. In: FELDMAN, B.F.; ZINKL, J.G.; JAIN, N.C. Schalm's veterinary hematology. 5.ed. Philadelphia: Lippincott Williams \& Wilkins, 2000. p. 733-739.
NAOM, P.C. Avanços tecnológicos em hematologia laboratorial. Revista Brasileira de Hematologia e Hemoterapia, São José do Rio Preto, v. 23, n. 2, p. 111-119, 2001.

OGILVIE, G.K., MOORE, A.S. Paraneoplastic syndromes. In: MANAGING the veterinary cancer pantient. Trenton: Veterinary Learning Systems, 1997. p. 197-222.

PERKINS, P. Hematologic abnormalities accompanying leukemia. In: FELDMAN, B.F.; ZINKL, J.G.; JAIN, N.C. Schalm's veterinary hematology. 5.ed. Philadelphia: Lippincott Williams \& Wilkins, 2000. p. 740-746.

SHARKEY, L.C.; ROSOL, T.J.; GRÖNE, A.; WARD, H.; STEINMEYER,C. Production of granulocyte colony-stimulating factor and granulocytemacrophage colony-stimulating factor by carcinomas in a dog and a cat with paraneoplastic leukocytosis. Journal of Veterinary Internal Medicine, Lawrence, v. 10, p. 405-408, 1996.

STOCKHAM, S.L.; KEETON, K.S.; SZLADOVITS,B. Clinical assesment of leukocytosis: distinguishing leukocytoses caused by inflammatory, glucocorticoid, physiologic, and leukemic disorders or conditions. The Veterinary Clinics Small Animal Practice, Orlando, v. 33, p. 1335-1357, 2003.

SQUIRES, R.A; THOMPSON, K.G. Extreme neutrophilic leucocytosis and intrathoracic mass in a Fox Terrier. New Zealand Veterinary Journal, Palmerston North, v. 52, n. 1, p. 50-51.2004.

THOMPSON, J.P., CHRISTOPHER, M.M., ELLISON, G.W., HOMER, B.L., BUCHANAN, B.A. Paraneoplastic leukocytosis associated with a rectal adenomatous polyp in a dog. ). Journal of the American Veterinary Medical Association, Schaumburg, v. 201, n. 5 p. 737-738, 1992.

YOUNG,K.M. Hemolymphatic system: Introduction. In: MORGAN, R.V. Handbook of Small Animal Practice. 3.ed. Philadelphia: W.B. Saunders, 1997. p. 653-655.

Recebido para publicação: 30/06/2005

Aprovado:

$20 / 09 / 2005$ 